\title{
Linagliptin-Associated Alopecia and Bullous Pemphigoid
}

\author{
Ali Someili ${ }^{1}$, Khalid Azzam ${ }^{1}$, Mohannad Abu Hilal ${ }^{2}$ \\ ${ }^{1}$ Department of Medicine, McMaster University, Hamilton, ON, Canada \\ ${ }^{2}$ Department of Dermatology, McMaster University, Hamilton, ON, Canada
}

Received: 13/07/2019

Accepted: $31 / 07 / 2019$

Published: 10/09/2019

How to cite this article: Someili A, Azzam K, Abu Hilal M. Linagliptin-associated alopecia and bullous pemphigoid. EJCRIM 2019;6:

doi:10.12890/2019_001207.

Conflicts of Interests: The Authors declare that there are no competing interest

This article is licensed under a Commons Attribution Non-Commercial 4.0 License

\section{ABSTRACT}

Bullous pemphigoid is a chronic autoimmune blistering disease. Recently, several reports suggested dipeptidyl peptidase 4 (DPP-4) inhibitors, also known as gliptins, were a potential cause of drug-induced bullous pemphigoid but not of both bullous pemphigoid and alopecia areata together. Here we describe the case of a 68-year-old man with type 2 diabetes mellitus who developed new onset diffuse alopecia on the scalp with diffuse tense bullae over his body a few months after linagliptin was introduced for better control of his diabetes.

DPP-4 inhibitors are not known to increase the risk of alopecia. To the best of our knowledge, this is the first report of linagliptin-associated alopecia areata and bullous pemphigoid, which may help demonstrate if there are any links between DPP-4 inhibitors and alopecia.

\section{LEARNING POINTS}

- This is the first report of linagliptin-associated alopecia areata and bullous pemphigoid (BP), which may help demonstrate a link between DPP-4 inhibitors and alopecia.

- Since the time of onset of BP after initiation of a DPP-4 inhibitor varies, a high index of suspicion is needed for diagnosis.

- Early diagnosis is essential as DPP-4 inhibitor withdrawal has a significant effect on disease remission.

\section{KEYWORDS}

Gliptin, drug, bullous pemphigoid

\section{INTRODUCTION}

Bullous pemphigoid (BP) is an autoimmune disease where autoantibodies target structural proteins at the dermal-epidermal junction. Two hemidesmosomal proteins, $230 \mathrm{kDa}$ protein and $180 \mathrm{kDa}$ antigen, have been identified as the major targets of BP autoantibodies. $\mathrm{BP}$ manifests with tense blisters on the skin $^{[1]}$. It is poorly understood although many trigger factors have been identified, such as contrast material injection, surgical procedures, mechanical trauma, insect bites, thermal burns, radiotherapy and ultraviolet exposure associated with pre-existing psoriasis ${ }^{[2]}$. Linagliptin is one of the new dipeptidyl peptidase-4 (DPP-4) inhibitors used in the treatment of type 2 diabetes mellitus (DM). DPP-4 inhibitors have been recently implicated in inducing BP, but the mechanism is not entirely clear. DPP-4 inhibitors may induce anti-basement membrane zone antibodies or other structurally similar antibodies, leading to sub-epidermal bullae and BP ${ }^{[3]}$. Many recent case reports show that use of DPP-4 inhibitors is a risk factor for BP onset, but there is no evidence of an association with alopecia.

\section{CASE DESCRIPTION}

A 68-year-old Caucasian man with a complex medical history including type 2 DM presented to the emergency department with a 3-4-week history of generalized pruritus, new onset diffuse alopecia and diffuse bullae over his trunk, arms and legs. The patient initially had developed bullae and blisters over his legs. Simultaneously, he noticed a significant loss of his scalp and beard hair as well as his eyebrows. This was 
accompanied by intense pruritus over the abdomen and back for approximately 2 weeks prior to the development of the bullae. The intense itching, development of further bullae, and almost complete alopecia prompted the patient to present to the emergency department.

A review of his history did not reveal any drug allergies and he denied a family history of autoimmune conditions. He had not travelled anywhere recently and did not present with any constitutional symptoms or myalgias. His home medications included linagliptin, allopurinol, amlodipine, atorvastatin, furosemide, hydralazine, levothyroxine, pantoprazole, rivaroxaban, terazosin and insulin.

His vital signs were all within normal limits: he was afebrile at $36.8^{\circ} \mathrm{C}$, his heart rate was $59 \mathrm{bpm}$, blood pressure was $118 / 73 \mathrm{mmHg}$, and oxygen saturation was $98 \%$ on room air. Physical examination revealed bullae over his back, abdomen and both lower legs and measuring approximately $1-3 \mathrm{~cm}$ in diameter (Fig. 1). He also had numerous smaller bullae over both flanks, upper arms and lower legs and measuring approximately $0.5-1 \mathrm{~cm}$ in diameter. There was no specific dermatomal distribution and no oral ulcerations or mucositis. The patient had diffuse alopecia on his scalp, eyebrows and beard. There was no erythema, scaling or scarring associated with the hair loss (Fig. 2).

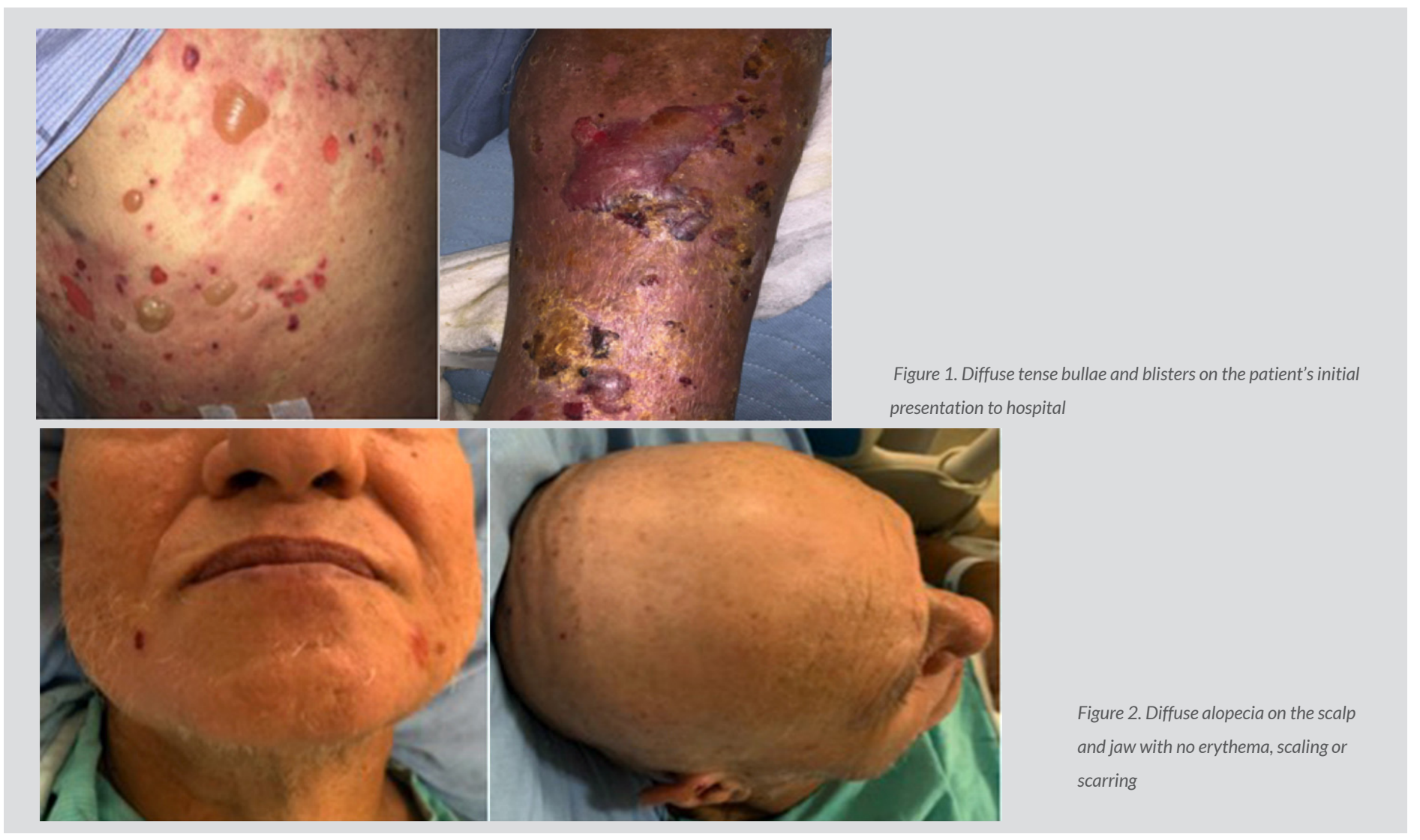

Investigations

Initial laboratory investigations revealed an elevated creatinine level of $300 \mathrm{mmol} / \mathrm{l}$ (baseline in the mid-200s) with normal electrolytes. The patient also had an elevated CRP level of $12.6 \mathrm{mg} / \mathrm{l}$, his white blood cell count was normal at $10.3 \mathrm{~g} / \mathrm{l}$, and haemoglobin concentration was $110 \mathrm{~g} / \mathrm{l}$.

Hospital course

Skin biopsies performed on admission showed sub-epidermal blisters with multiple eosinophils highly suggestive of BP (Fig. 3). Eosinophils are commonly seen in BP, but the diffuse eosinophilia seen in the biopsy specimen raised the possibility of drug-induced BP. Direct immunofluorescence showed deposition of IgG and C3 along the basement membrane confirming BP (Fig. 4). Interestingly, linagliptin had been introduced a few months before the onset of cutaneous eruptions. At that point, linagliptin was highly suspected as the cause of BP and alopecia and therefore was discontinued. The patient was started on prednisone $40 \mathrm{mg}$ with significant improvement of his skin lesions. He was then followed by the dermatology department and was started on mycophenolic acid (720 mg by mouth twice a day as a steroidsparing agent) and topical clobetasol cream. At his 6-month follow-up, 80-90\% of his skin lesions had resolved and his hair had grown back (Fig. 5A,B). 


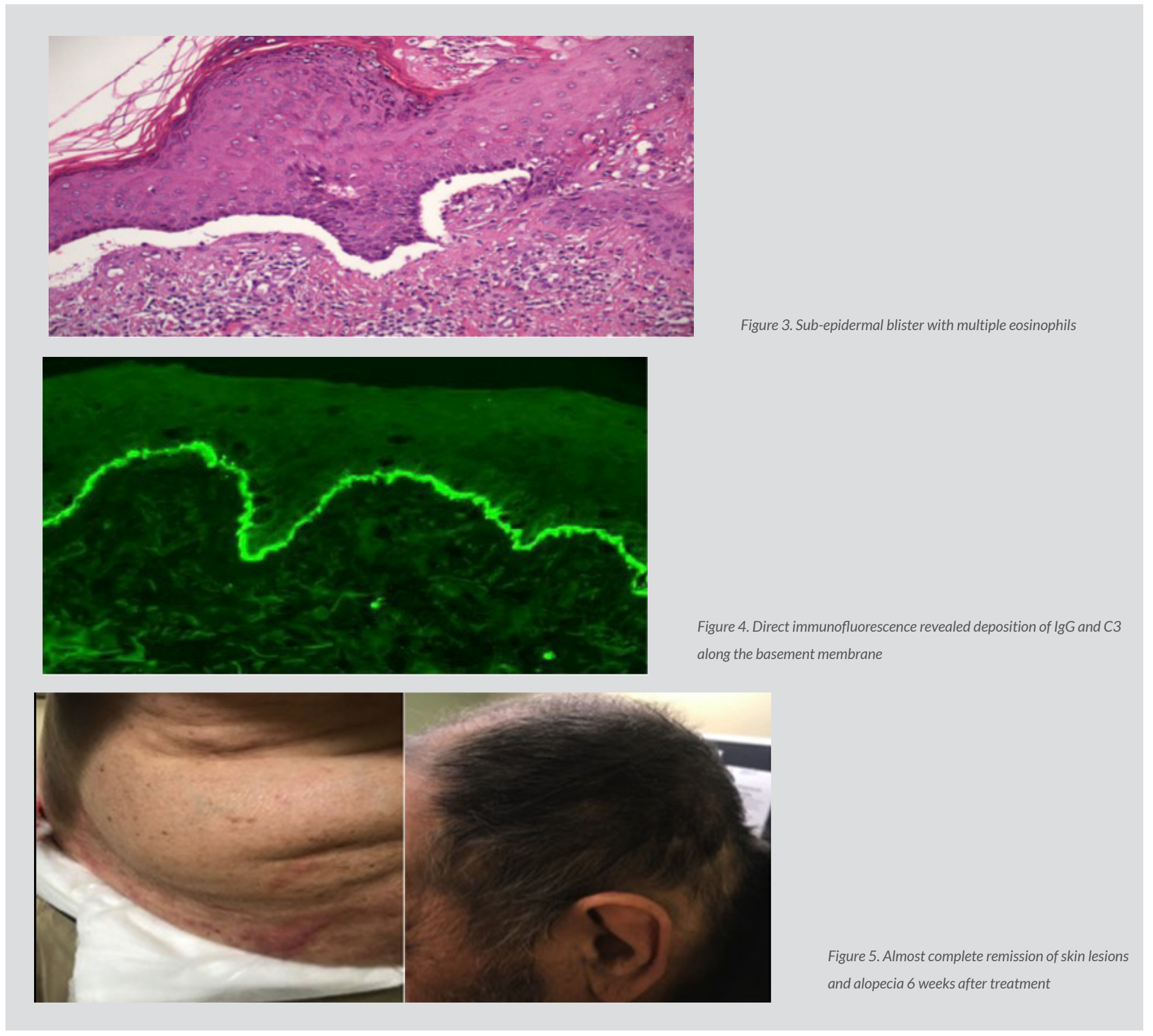

\section{DISCUSSION}

BP is not uncommon and has an incidence of $0.2-3 / 100,000$ person-years with a higher incidence in older age groups. A UK study estimated the incidence there was $1.4 / 100,000$ person-years ${ }^{[4]}$. There is a wide variation in mortality rate, with a 1-year mortality rate of $13-41 \%$ in Europe and $11-23 \%$ in the USA ${ }^{[5]}$. The classic manifestation of BP is diffuse tense blisters, but clinical presentation can also include diffuse urticarial or dermatitis plaques ${ }^{[6]}$.

DPP-4 inhibitors are a class of oral hypoglycaemic agents which can be used to treat type 2 DM. DPP-4 inhibitors work by increasing glucagon-like peptide-1 and glucose-dependent insulin-trophic polypeptide which leads to increased insulin and inhibits glucagon. Sitagliptin was the first agent of this class to be approved by the FDA in 2006 $6^{[7]}$. The DPP-4 enzyme has many functions including biological roles in pro-inflammatory pathways ${ }^{[8]}$. DPP- 4 is also known as CD26 and is widely expressed in various cell types throughout the body including the skin, but the role of the DPP-4 enzyme in autoimmune pathogenesis has not yet been clearly elucidated ${ }^{[9,10]}$. A large observational study has indicated that the use of DPP-4 inhibitors is associated with an overall $75 \%$ increase in the risk of inflammatory bowel disease in patients with type $2 \mathrm{DM}^{[9]}$. However, another large observational study indicated that DPP-4 inhibitor combination therapy appears to be associated 
with a decreased risk of autoimmune diseases, including rheumatoid arthritis, compared with non-DPP-4 inhibitor combination therapy ${ }^{[11]}$. A few months after starting linagliptin, our patient developed alopecia areata totalis and a skin eruption shown to be BP by histological examination and immunofluorescence. Several cases of linagliptin-induced BP have been reported (Table 1). However, none of those cases described both alopecia areata and BP simultaneously due to linagliptin.

\section{CONCLUSION}

This is the first report of linagliptin-associated alopecia areata and BP and may indicate a link between DPP-4 inhibitors and alopecia. Although DPP-4 inhibitors are known to be associated with BP, the pathogenesis is still not completely understood. Early diagnosis is essential as agent withdrawal has a significant effect on disease remission.

\begin{tabular}{|c|c|c|c|c|c|c|}
\hline Author & $\begin{array}{l}\text { Patient age } \\
\text { and gender }\end{array}$ & $D P P-4 i$ & $\begin{array}{l}\text { Length of } \\
\text { time before } \\
\text { BP onset }\end{array}$ & Clinical presentation & Treatment & Outcome \\
\hline \multirow[t]{3}{*}{ Mendonça et al. ${ }^{[12]}$} & $82 M$ & Linagliptin & 45 Days & $\begin{array}{l}\text { Pruritic cutaneous eruption. } \\
\text { No mucosal involvement }\end{array}$ & $\begin{array}{l}\text { Prednisone taper over } 6 \text { months } \\
\text { and etamethasone-gentamicin } \\
\text { cream }\end{array}$ & $\begin{array}{l}\text { Followed for } 6 \text { months, no further } \\
\text { exacerbations following linagliptin } \\
\text { withdrawal }\end{array}$ \\
\hline & $77 F$ & $\begin{array}{l}\text { Vildagliptin/ } \\
\text { metformin }\end{array}$ & $N A$ & $\begin{array}{l}\text { Mucosal involvement at } \\
\text { onset; afterwards, mucosal } \\
\text { and cutaneous }\end{array}$ & Prednisone $1 \mathrm{mg} / \mathrm{kg} /$ day & Lost to follow-up \\
\hline & $72 \mathrm{~F}$ & $\begin{array}{l}\text { Vildagliptin/ } \\
\text { metformin }\end{array}$ & 3 Months & $\begin{array}{l}\text { Pruriginous tense bullae over } \\
\text { a urticarial base. } \\
\text { No mucosal involvement }\end{array}$ & Prednisone $1 \mathrm{mg} / \mathrm{kg} /$ day & $\begin{array}{l}\text { Followed for }>8 \text { months, no further } \\
\text { exacerbation }\end{array}$ \\
\hline Sakai et al. ${ }^{[13]}$ & $76 F$ & Linagliptin & 9 Months & $\begin{array}{l}\text { Tense bullae over whole body. } \\
\text { No mucosal involvement }\end{array}$ & $\begin{array}{l}\text { Topical dexamethasone valerate; } \\
\text { minocycline } 100 \mathrm{mg} \text { daily was } \\
\text { added for a while }\end{array}$ & $\begin{array}{l}\text { Complete remission was achieved at } \\
16 \text { weeks after the discontinuation } \\
\text { of linagliptin }\end{array}$ \\
\hline \multirow[t]{2}{*}{ Haber et al. ${ }^{[3]}$} & $60 \mathrm{M}$ & Linagliptin & 4 Months & $\begin{array}{l}\text { Pruritus and erythematous } \\
\text { tense bullae on the limbs. } \\
\text { No mucosal involvement }\end{array}$ & Topical corticosteroid & $\begin{array}{l}\text { No clinical recurrence of BP during } 3 \\
\text { months of follow-up }\end{array}$ \\
\hline & $70 \mathrm{~F}$ & Linagliptin & 3 Months & $\begin{array}{l}\text { Pruritus and tense bullae on } \\
\text { the trunk. } \\
\text { No mucosal involvement }\end{array}$ & Topical clobetasol propionate & $\begin{array}{l}\text { Followed for } 5 \text { months with no } \\
\text { clinical recurrence of } B P\end{array}$ \\
\hline 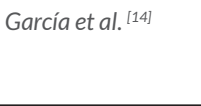 & $74 F$ & $\begin{array}{l}\text { Vildagliptin/ } \\
\text { metformin }\end{array}$ & 12 Months & $\begin{array}{l}\text { Pruritic bullous skin lesions on } \\
\text { the trunk. } \\
\text { No mucosal involvement }\end{array}$ & $\begin{array}{l}\text { Oral prednisone for } 3 \\
\text { weeks+topical clobetasol }\end{array}$ & $\begin{array}{l}\text { No clinical recurrence after } 3 \text { years } \\
\text { of follow-up }\end{array}$ \\
\hline \multirow[t]{5}{*}{ Yoshiji et al. ${ }^{[15]}$} & $81 \mathrm{M}$ & Linagliptin & 9 Months & $\begin{array}{l}\text { Erythematous tense bullae } \\
\text { over entire body. } \\
\text { No mucosal involvement }\end{array}$ & $\begin{array}{l}20 \mathrm{mg} \text { prednisone, then tapered } \\
\text { off }\end{array}$ & $\begin{array}{l}\text { Complete remission } 6 \text { weeks after } \\
\text { agent withdrawal }\end{array}$ \\
\hline & $86 M$ & Linagliptin & 9 Months & $\begin{array}{l}\text { Erythematous tense bullae } \\
\text { over entire body. } \\
\text { No mucosal involvement }\end{array}$ & $\begin{array}{l}\text { Started on } 20 \quad \mathrm{mg} / \text { day } \\
\text { prednisolone, which was tapered } \\
\text { to } 2 \mathrm{mg} / \text { day over } 10 \text { months }\end{array}$ & $\begin{array}{l}\text { Complete remission } 4 \text { weeks after } \\
\text { agent withdrawal }\end{array}$ \\
\hline & $83 F$ & $\begin{array}{l}\text { Linagliptin, } \\
\text { sitagliptin }\end{array}$ & 15 Months & $\begin{array}{l}\text { Erythematous tense bullae. } \\
\text { No mucosal involvement }\end{array}$ & $\begin{array}{l}\text { Prednisolone ( } 15 \mathrm{mg} / \text { day), then } \\
\text { replaced by IVIG after } 3 \text { days } \\
\text { because of poor control of BP }\end{array}$ & $\begin{array}{l}\text { Complete remission } 2 \text { weeks after } \\
\text { agent withdrawal }\end{array}$ \\
\hline & $86 \mathrm{~F}$ & Vildagliptin & 6 Months & $\begin{array}{l}\text { Erythematous tense bullae. } \\
\text { No mucosal involvement }\end{array}$ & $\begin{array}{l}\text { Started on } 40 \quad \mathrm{mg} / \text { day } \\
\text { prednisolone and then received } \\
\text { IVIG due to poor control of skin } \\
\text { symptoms }\end{array}$ & $\begin{array}{l}\text { Complete remission } 4 \text { weeks after } \\
\text { agent withdrawal }\end{array}$ \\
\hline & $63 \mathrm{M}$ & Anagliptin & 5 Months & $\begin{array}{l}\text { Erythematous bullous } \\
\text { eruptions over entire body. No } \\
\text { mucosal involvement }\end{array}$ & $\begin{array}{l}\text { Prednisolone (20 mg/day), } \\
\text { tapered and stopped within } 14 \\
\text { days }\end{array}$ & $\begin{array}{l}\text { Complete remission } 2 \text { weeks after } \\
\text { agent withdrawal }\end{array}$ \\
\hline Guliani et al. ${ }^{[16]}$ & $69 \mathrm{M}$ & $\begin{array}{l}\text { Teneligliptin/ } \\
\text { metformin }\end{array}$ & 4 Months & $\begin{array}{l}\text { Erythematous, itchy, fluid- } \\
\text { filled lesions. No mucosal } \\
\text { involvement }\end{array}$ & Topical clobetasol propionate & $\begin{array}{l}\text { The skin lesions improved in a } \\
\text { month. } \\
\text { Duration of follow-up unknown }\end{array}$ \\
\hline
\end{tabular}




\begin{tabular}{|c|c|c|c|c|c|c|}
\hline Author & $\begin{array}{l}\text { Patient age } \\
\text { and gender }\end{array}$ & DPP-4i & $\begin{array}{l}\text { Length of } \\
\text { time before } \\
\text { BP onset }\end{array}$ & Clinical presentation & Treatment & Outcome \\
\hline Takama et al. ${ }^{[17]}$ & $86 F$ & $\begin{array}{r}\text { Linagliptin, } \\
\text { anagliptin }\end{array}$ & 6 Weeks & $\begin{array}{l}\text { Multiple blisters on both legs. } \\
\text { No mucosal nvolvement }\end{array}$ & $\begin{array}{l}\text { Initially topical clobetasol } \\
\text { propionate. On day } 185, \mathrm{BP} \\
\text { relapsed so prednisone } 7.5 \mathrm{mg} \\
\text { and mizoribine started }\end{array}$ & $\begin{array}{l}\text { Complete remission on day } 220 . \\
\text { Then no relapses up to day } 297\end{array}$ \\
\hline Maki et al. ${ }^{[18]}$ & $70 \mathrm{M}$ & Teneligliptin & 4 Weeks & $\begin{array}{l}\text { Multiple blisters on the trunk. } \\
\text { No mucosal involvement }\end{array}$ & None & $\begin{array}{l}\text { Followed up for } 2 \text { months with no } \\
\text { clinical recurrence of BP }\end{array}$ \\
\hline Harada et al. ${ }^{[19]}$ & $78 \mathrm{M}$ & Sitagliptin & 3 Years & $\begin{array}{l}\text { Cutaneous blisters on the } \\
\text { bilateral limbs and abdomen }\end{array}$ & Prednisolone $20 \mathrm{mg}$ & Died on day 14 \\
\hline Esposito et al. ${ }^{[20]}$ & $73 F$ & Linagliptin & 5 Months & Diffuse bullous rash & $\begin{array}{l}\text { IV methylprednisolone }(1 \mathrm{mg} / \mathrm{kg} / \\
\text { day for } 10 \text { days, then tapered) } \\
\text { and azathioprine }(100 \mathrm{mg} / \text { day }) \\
\text { for } 12 \text { weeks }\end{array}$ & Still in remission at 1-year follow-up \\
\hline Harada et al. ${ }^{[19]}$ & $78 \mathrm{M}$ & Sitagliptin & 3 Years & $\begin{array}{l}\text { Cutaneous blisters on the } \\
\text { bilateral limbs and abdomen }\end{array}$ & Prednisolone $20 \mathrm{mg}$ & Died on day 14 \\
\hline Esposito et al. ${ }^{[20]}$ & $73 F$ & Linagliptin & 5 Months & Diffuse bullous rash & $\begin{array}{l}\text { IV methylprednisolone }(1 \mathrm{mg} / \mathrm{kg} / \\
\text { day for } 10 \text { days, then tapered) } \\
\text { and azathioprine }(100 \mathrm{mg} / \text { day }) \\
\text { for } 12 \text { weeks }\end{array}$ & Still in remission at 1-year follow-up \\
\hline Keseroglu et al. ${ }^{[21]}$ & $61 \mathrm{~F}$ & $\begin{array}{l}\text { Vildagliptin/ } \\
\text { metformin }\end{array}$ & 10 Months & $\begin{array}{l}\text { Pruritic vesiculobullous } \\
\text { lesions. No mucosal } \\
\text { involvement }\end{array}$ & Topical clobetasol & $\begin{array}{l}\text { Remission within } 3 \text { weeks. } \\
\text { No further follow-up mentioned }\end{array}$ \\
\hline \multirow[t]{3}{*}{ Béné et al. ${ }^{[22]}$} & $86 F$ & $\begin{array}{l}\text { Vildagliptin/ } \\
\text { metformin }\end{array}$ & 2 Months & $\begin{array}{l}\text { Erythematous bullous } \\
\text { eruption, with bullae on } \\
\text { healthy skin and extensive } \\
\text { erosions on the body }\end{array}$ & Topical clobetasol & $\begin{array}{l}\text { Condition improved after } \\
\text { withdrawal of vildagliptin. } \\
\text { Duration of follow-up not mentioned }\end{array}$ \\
\hline & $79 \mathrm{M}$ & $\begin{array}{l}\text { Vildagliptin/ } \\
\text { metformin }\end{array}$ & 37 Months & $\begin{array}{l}\text { Generalized erythematous } \\
\text { vesicular bullous eruption }\end{array}$ & Topical clobetasol & $\begin{array}{l}\text { The lesions recurred at } 3 \text { months but } \\
\text { improved after discontinuation of } \\
\text { vildagliptin. } \\
\text { The duration of follow-up not } \\
\text { mentioned }\end{array}$ \\
\hline & $77 F$ & Vildagliptin & 26 Months & Pruriginous bullous eruption & Topical clobetasol & $\begin{array}{l}\text { Condition improved after } \\
\text { withdrawal of vildagliptin. } \\
\text { The duration of follow up not } \\
\text { mentioned }\end{array}$ \\
\hline Aouidad et al. ${ }^{[23]}$ & $61 \mathrm{M}$ & $\begin{array}{l}\text { Vildagliptin/ } \\
\text { metformin }\end{array}$ & 5 Months & $\begin{array}{l}\text { Pruritus and bullous } \\
\text { haemorrhagic lesions over } \\
\text { an erythematous base } \\
\text { disseminated on the trunk and } \\
\text { the limb }\end{array}$ & Topical corticosteroid & $\begin{array}{l}\text { Complete remission } 2 \text { weeks after } \\
\text { agent withdrawal }\end{array}$ \\
\hline \multirow[t]{3}{*}{ Pasmatzi et al. ${ }^{[24]}$} & $59 F$ & $\begin{array}{l}\text { Vildagliptin/ } \\
\text { metformin }\end{array}$ & 2 Months & $\begin{array}{l}\text { Diffuse bullous eruption } \\
\text { mostly on an erythematous } \\
\text { base }\end{array}$ & $\begin{array}{l}0.5 \mathrm{mg} / \mathrm{kg} / \text { day } \\
\text { methylprednisolone which was } \\
\text { tapered over } 8 \text { weeks }\end{array}$ & $\begin{array}{l}\text { Complete remission } 10 \text { weeks after } \\
\text { agent withdrawal }\end{array}$ \\
\hline & $67 \mathrm{M}$ & $\begin{array}{l}\text { Vildagliptin/ } \\
\text { metformin }\end{array}$ & 2 Months & $\begin{array}{l}\text { Diffuse bullous eruption } \\
\text { mostly on an erythematous } \\
\text { base }\end{array}$ & $\begin{array}{l}200 \mathrm{mg} / \text { day doxycycline } \\
\text { for } 4 \text { weeks }\end{array}$ & $\begin{array}{l}\text { Complete remission } 8 \text { weeks after } \\
\text { agent withdrawal }\end{array}$ \\
\hline & $64 \mathrm{M}$ & Linagliptin & 6 Months & $\begin{array}{l}\text { Cutaneous and severe } \\
\text { mucosal involvement }\end{array}$ & $0.3 \mathrm{mg} / \mathrm{kg} /$ day oral prednisolone & $\begin{array}{l}\text { Improvement of only cutaneous } \\
\text { lesions } 2 \text { weeks after agent } \\
\text { withdrawal. } \\
\text { Mucosal lesions improved } 2 \text { weeks } \\
\text { after starting prednisone. No further } \\
\text { follow-up }\end{array}$ \\
\hline
\end{tabular}




\section{REFERENCES}

1. Meyer N, Misery L. Geoepidemiology considerations of auto-immune pemphigus. Autoimmun Rev 2009;9(5):A379-382

2. Dănescu S, Chiorean R, Macovei V, Sitaru C, Baican A. Role of physical factors in the pathogenesis of bullous pemphigoid: case report series and a comprehensive review of the published work. J Dermatol 2016;43(2):134-140.

3. Haber R, Fayad AM, Stephan F, Obeid G, Tomb R. Bullous pemphigoid associated with linagliptin treatment. JAMA Dermatol 2016;152(2):224-226.

4. Brick K, Weaver C, Lohse C, Pittelkow M, Lehman J, Camilleri M, et al. Incidence and mortality rates of bullous pemphigoid in Olmsted County, Minnesota, over 6 decades. J Am Acad Dermatol 2014;71(1):92-99.

5. Langan SM, Smeeth L, Hubbard R, Fleming KM, Smith CJP, West J. Bullous pemphigoid and pemphigus vulgaris-incidence and mortality in the UK: population based cohort study. BMJ 2008;337(7662):160-163.

6. Zhao CY, Murrell DF. Advances in understanding and managing bullous pemphigoid. F1000Res 2015;4. doi: 10.12688/f1000research.6896.1

7. Aovi FI, Halder SS. A study on the prescribing patterns of dipeptidyl peptidase 4 inhibitors in a multidisciplinary hospital complex Birdem, Bangladesh. IOSR JEF 2016;1(10):1013.

8. da Silva Júnior WS, de Godoy-Matos AF, Kraemer-Aguiar LG. Dipeptidyl peptidase 4: a new link between diabetes mellitus and atherosclerosis? Biomed Res Int 2015;2015:110.

9. Kim S, Schneeweiss S, Glynn RJ, Doherty M, Goldfine AB, Solomon DH. Dipeptidyl peptidase-4 inhibitors in type 2 diabetes may reduce the risk of autoimmune diseases: a population-based cohort study. Ann Rheum Dis 2016;74(11):1968-1975.

10. Attaway A, Mersfelder TL, Vaishnav S, Baker JK. Bullous pemphigoid associated with dipeptidyl peptidase IV inhibitors. A case report and review of literature. J Dermatol Case Rep 2014;8(1):24-28.

11. Abrahami D, Douros A, Yin H, Hoi Yun Yu O, Renoux C, Bitton A, et al. Dipeptidyl peptidase-4 inhibitors and incidence of inflammatory bowel disease among patients with type 2 diabetes: population based cohort study. BMJ 2018;360:k872.

12. Mendonça FMI, Martín-Gutierrez FJ, Ríos-Martín JJ, Camacho-Martinez F. Three cases of bullous pemphigoid associated with dipeptidyl peptidase-4 inhibitors - one due to linagliptin. Dermatology 2016;232(2):249-253.

13. Sakai A, Shimomura Y, Ansai O, Saito Y, Tomii K, Tsuchida Y, et al. Linagligiptin-associated bullous pemphigoid that was most likely caused by IgG autoantibodies against the mid portion of BP180. Br J Dermatol 2017;176(2):541-543.

14. García M, Aranburu MA, Palacios-Zabalza I, Lertxundi U, Aguirre C. Dipeptidyl peptidase-IV inhibitors induced bullous pemphigoid: a case report and analysis of cases reported in the European pharmacovigilance database. J Clin Pharm Ther 2016;41(3):368-370.

15. Yoshiji S, Murakami T, Harashima SI, Ko R, Kashima R, Yabe D, et al. Bullous pemphigoid associated with dipeptidyl peptidase-4 inhibitors: A report of five cases. J Diabetes Investig 2018;9(2):445-447.

16. Guliani A, Bishnoi A, Aggarwal D, Parsad D. Teneligliptin-associated bullous pemphigoid in an elderly man with diabetes. Postgrad Med J 2018;94(1117):662-663.

17. Takama H, Yoshida M, Izumi K, Yanagishista T, Muto J, Ohshima Y, et al. Dipeptidyl peptidase-4 inhibitor-associated bullous pemphigoid: recurrence with epitope spreading. Acta Derm Venereol 2018;98(10):983-984.

18. Maki N, Nishie W, Takazawa M, Kakurai M, Yamada T, Umemoto N, et al. Dipeptidyl peptidase-4 inhibitor-associated bullous pemphigoid in a patient with acquired reactive perforating collagenosis. J Dermatol 2018;45(5):600-602.

19. Harada M, Yoneda A, Haruyama S, Yabuki K, Honma Y, Hiura M, et al. Bullous pemphigoid associated with the dipeptidyl peptidase-4 inhibitor sitagliptin in a patient with liver cirrhosis complicated with rapidly progressive hepatocellular carcinoma. Intern Med 2017;56(18):2471-2474.

20. Esposito I, Moretta G, Peris K, De Simone C. Linagliptin-induced bullous pemphigoid. Int J Dermatol 2017;56(12):1467-1469.

21. Keseroglu HO, Taş-Aygar G, Gönül M, Gököz O, Ersoy-Evans S. A case of bullous pemphigoid ınduced by vildagliptin. Cutan Ocul Toxicol $2017 ; 36(2): 201-202$.

22. Béné J, Jacobsoone A, Coupe P, Auffret M, Babai S, Hillaire-Buys D, et al. Bullous pemphigoid induced by vildagliptin: a report of three cases. Fundam Clin Pharmacol 2015;29(1):112-114.

23. Aouidad I, Fite C, Marinho E, Deschamps L, Crickx B, Descamps V. A case report of bullous pemphigoid induced by dipeptidyl peptidase-4 inhibitors. JAMA Dermatol 2013;149(2):243-244.

24. Pasmatzi E, Monastirli A, Habeos J, Georgiou S, Tsambaos D. Dipeptidyl peptidase-4 inhibitors cause bullous pemphigoid in diabetic patients: report of two cases. Diabetes Care 2011;34(8):e133-e133. 